\title{
The Frobenius Method for Solving Ordinary Differential Equation with Coefficient Variable
}

\author{
Anil Hakim Syofra ${ }^{1}$, Rika Permatasari ${ }^{2}$, Lily Adriani Nazara ${ }^{3}$ \\ Student of Faculty of Mathematics and Natural Science, University of Sumatera Utara, Indonesia
}

\begin{abstract}
One of the problems in differential equation is to get solution of Ordinary differential equation with coefficient variable. So that needed a method to solve it, it is the series solution. The series solution at point $t$ can be used if $t=0$ is ordinary point of ordinary differential equations, but if $t=0$ is singular point, it is needed an extend series solution which called Frobenius method. This research aims to determine the form of extend series solution at singular points and the form of linear independently second solutions. The results of this research indicate the form of extend series solution at singular point and then based on roots of indicial terms, there are three kinds linear independently second solution where distinct roots not differing by an integer, roots differing by an integer, and double root.
\end{abstract}

Keywords: Ordinary Differential Equation, Ordinary Points, Singular Points, Series Solution, Frobenius Method

\section{Introduction}

Ordinary differential equation is a differential equation that includes the derivative of a function with one of dependent variable. In discussing an ordinary differential equation, can not be separated in the study of finding a solution of the differential equations. The Solutions of differential equations orde- $n$ is a function that satisfies the differential equation.

There are many ways in solving ordinary differential equations, but frequent obstacles in obtaining the solution of differential equations, especially the differential equation that has the form of a variable coefficient. Variable coefficient differential equations which have special shapes such as differential equations Cauchy-Euler-Lagrange differential equations and Legendre, can be solved by simplifying the form of the differential equation. However, if the coefficient of variables do not have a particular form it will be difficult to obtain the differential equation solution.

The connection for the completion of differential equations developed a method that can resolve the variable coefficient differential equations. The method can be used to declare the settlement in the form of power series solutions. This method is very efficient to use the variable coefficient differential equation, because the coefficients of the differential equations are not required to have a certain shape.

Completion of differential equations using power series solution keanalitikan attention coefficients of a differential equation. A function $f(t)$ is said to be analytic at $t_{0}$ if $f$ $(t)$ has a form of the series:

$$
f(t)=\sum_{n=0}^{\infty} \frac{f^{(n)}\left(t_{0}\right)}{n !}\left(t-t_{0}\right)^{n}
$$

If a differential equation is represented in the form $y^{\prime \prime}+$ $P(x) y^{\prime}+Q(x) y=0$ with $P(x)$ and $Q(x)$ analytic at $x=$ $x_{0}$, then the solution of differential equation $y(\mathrm{x})$ will be analytic at $x=x_{0}$ differential equation means that the solution can be represented in the form:

$$
y(x)=\sum_{m=0}^{\infty} a_{m}\left(x-x_{0}\right)^{m}
$$

However, if $P(x)$ and $Q(x)$ is not analytic at $x=x_{0}$, in other words have a singular point at $x=x_{0}$ then the solution $y(x)$ can not be represented in the series so we need a method of power series expanded or known as the Frobenius method.

This method has at least one solution in the form:

$$
y(x)=\left(x-x_{0}\right)^{r} \sum_{m=0}^{\infty} a_{m}\left(x-x_{0}\right)^{m}
$$

Where $r$ is the root of the equation indicator differential equations. In completing the second-order differential equation. the solution will be sought both from differential equations, which form the second solution of differential equations depend on the roots of the indicator, which is where the roots may be the same or different, wherein the solution contains a logarithmic function.

Frobenius was very efficient method used to find the solution of differential equations with coefficients in the form of function.

Frobenius method is widely used in the search for a solution from the application of differential equations, including Bessel equation, deployment the temperature in the tube, Laguerre equation used in quantum mechanics of the hydrogen atom, and hypergeometric of Gauss equation.

\section{Method}

This research is basic or theoretical. The method used is descriptive method, by analyzing the theories that are 


\section{International Journal of Science and Research (IJSR) \\ ISSN (Online): 2319-7064 \\ Index Copernicus Value (2013): 6.14 | Impact Factor (2015): 6.391}

relevant to the issues to be discussed, as well as based on the study of literature.

The steps of the research is:

1) Examine the theory of ordinary differential equations.

2) Examine theories about Ordinary points and singular points of ordinary differential equations.

3) Review the theory of power series solutions.

4) Reviewed series(1) Frobenius theorem.

5) Examine the roots of a second order differential equation indicator.

6) Examining the second row Frobenius theorem.

\section{Discussion of Results}

1) Forms Solution Expanded Powers series (Frobenius Method) at Singular Point

Differential Equations:

$$
t^{2} S^{\prime \prime}+t P(t) S^{\prime}+q(t) S=0
$$

$\mathrm{S}(\mathrm{t})=t^{x}$ is a solution of differential equations, will look for the solution of differential equations rows are mutually linearly independent by means letting,

where $U$ is a function $U(t)$.

$$
\mathrm{S}=\mathrm{U} \cdot t^{x}
$$

Lower the equation (2) to the second derivative and substituting into the differential equation (1).

Because $\mathrm{S}(\mathrm{t})=t^{x}$ is the solution of differential equations it is obtained :

$$
U^{\prime \prime} t^{x+2}+U^{\prime}\left(2 x t^{x+1}+P(t) t^{x+1}\right)=0
$$

with Integration

$$
\mathrm{U}=t^{-\left(P_{0}+2 x\right)+1}\left(k_{0}+k_{1} t+k_{2} t^{2}+. .\right)
$$

Since $\mathrm{S}=\mathrm{U} . t^{x}$ and refer to the equation indicators, which states the number of roots is $-\left(\mathrm{P}_{0}-1\right)$, then obtained:

$$
\mathrm{S}=t^{x}\left(k_{0}+k_{1} t+k_{2} t^{2}+. .\right)
$$

Thus obtained solution for the first series differential equation (1) which has a singular point at the point $\mathrm{t}=0$.

2) Both Forms Solution Differential Equation Coefficient Variables that Mutual Non Linear

Differential equations of the form:

$$
S^{\prime \prime}+\frac{p(t)}{t} S^{1}+\frac{\eta(t)}{t^{2}} S=0
$$

where $\mathrm{p}(\mathrm{t})$ and $\mathrm{q}(\mathrm{t})$ is analytic at $\mathrm{t}=0$, with $x_{1}>x_{2}$ where $x_{1}$ dan $x_{2}$ are the roots of the equation indicator

$$
x^{2}+\left(p_{0}-1\right) x+q_{0}=0 \text {. }
$$

will have the first solution that can be written in the form:

$$
S_{1}(t)=t^{x_{1}} \sum_{n=0}^{\infty} k_{n} t^{n}
$$

Has three cases form a second solution:
Case 1: The roots are different and the difference is not an integer.

In this case, $x_{1}-x_{2}=J^{*}$, with $\mathrm{J} *$ is not an integer

Letting:

With $\mathrm{W}$ is a function $\mathrm{W}(\mathrm{t})$.

$$
S_{2}=W \cdot S_{1}
$$

By using the same steps in the first problems, and $S_{1}$ is the solution of differential equations, it is obtained:

$$
t^{2} S_{1} W^{\prime \prime}+2 t^{2} S_{1}^{\prime} W^{\prime}+t p S_{1} W^{\prime}=0
$$

so that

$$
\frac{W^{\prime \prime}}{W^{\prime}}=-\left(\frac{2 x_{1}+p_{0}}{t}\right)+\sum_{n=0}^{\infty} \theta_{n} t^{n}
$$

In this case, the roots are different and the difference is not an integer then $2 x_{1}+P_{0}=1+J^{*}$, and of integration is obtained:

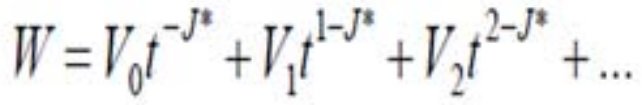

Because the $S_{2}=W \cdot S_{1}$, then obtained a second solution of differential equations are:

$$
S_{2}(t)=t^{x_{2}} \sum_{n=0}^{\infty} K_{n} t^{n}
$$

if the roots of equations of different indicators and the difference is not an integer.

Case 2: the difference of the roots is an integer, in this case, $x_{1}-x_{2}=J$, with $\mathrm{J}$ is an integer.

By using the same steps in proving the case 1, so that would be obtained by equation. In this case, the difference of the roots is an integer, so $2 x_{1}+P_{0}=1+J$ and of integration, is obtained:

$$
\begin{aligned}
W & =-\frac{1}{J} t^{-J}+v_{1} t^{1-J}+v_{2} t^{2-J}+\ldots+v_{J-1} t^{-1} \\
& +v_{J} \ln t+v_{J+1} t+v_{J+2} t^{2}+\ldots
\end{aligned}
$$

Because the $S_{2}=W \cdot S_{1}$, then obtained a second solution of differential equations are:

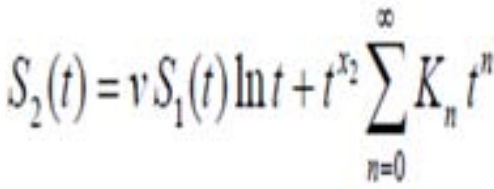

if the roots of different indicators and difference equations integers.

Case 3: twin roots, the roots of the equation Because the indicator has the same value that $x_{1}=x_{2}=x$, then the roots of the indicator $x=\frac{1}{2}\left(1-P_{0}\right)$. 


\section{International Journal of Science and Research (IJSR) \\ ISSN (Online): 2319-7064}

Index Copernicus Value (2013): 6.14 | Impact Factor (2015): 6.391

By using the same steps in proving the case (1), so that would be obtained equation. . Because $x=\frac{1}{2}\left(1-P_{0}\right)$ is the root of the root of the equation indicator, then $2 x+$ $P_{0}=1$, and of integration, is obtained:

$$
W=\ln t+v_{1} t+v_{2} t^{2}+v_{3} t^{3}+\ldots
$$

Because the $S_{2}=W \cdot S_{1}$, then obtained a second solution of differential equations are:

$$
S_{2}(t)=S_{1}(t) \ln t+t^{x+1} \sum_{n=0}^{\infty} K_{n} t^{n}
$$

if the roots of the same equation valuable indicators

\section{Conclusion}

From the discussion, it can be concluded that:

1) Forms Solution Expanded Powers series (Frobenius method) at Singular Point Any placement in the form of differential equations:

$$
S^{\prime \prime}+\frac{p(t)}{t} S^{\prime}+\frac{q(t)}{t^{2}} S=0
$$

where $\mathrm{p}(\mathrm{t})$ and $\mathrm{q}(\mathrm{t})$ analytic in $\mathrm{t}=0$, has at least one solution that can be written in the form:

$$
S(t)=t^{x} \sum_{n=0}^{\infty} k_{n} t^{n}
$$

where $\mathrm{x}$ is an indicator of the roots of equations differential equations.

\section{2) Both Forms Solution Variable Differential Equation} Coefficient of Mutual Non Linear

The second solution form of variable coefficient differential equations are mutually linearly independent with $x_{1}>x_{2}$ where $x_{1}$ and $x_{2}$ are the roots of the equation indicator $x^{2}+\left(P_{0}-1\right) x+q_{0}=0$, will have the first solution that can be written in form:

$$
S_{1}(t)=t^{x_{1}} \sum_{n=0}^{\infty} k_{n} t^{n}
$$

And has three cases form a second solution:

Case 1: The roots are different and the difference is not an integer.

$$
S_{2}(t)=t^{x_{2}} \sum_{n=0}^{\infty} K_{n} t^{n}
$$

Case 2: The second difference is an integer root

$$
S_{2}(t)=v S_{1}(t) \ln t+t^{x_{2}} \sum_{n=0}^{\infty} K_{n} t^{n},(t>0)
$$

Case 3: twin roots

$$
S_{2}(t)=S_{1}(t) \ln t+t^{x+1} \sum_{n=0}^{\infty} K_{n} t^{n},(t>0)
$$

With the $\mathrm{K} 1, \mathrm{~K} 2, \mathrm{~K} 3, \ldots . \mathrm{Kn}$ are constants.

\section{References}

[1] Boyce, William E. and Richard C. Di Prima.2001. Elementary Differential Equations and Boundary Value Problems. Edisi ke- USA: John Wiley \& Sons, Inc.

[2] Kohler, W. \& Lee Johnson. 2006 Elementary Differential Equations with Boundary Value Problems. Edisi ke-2. USA: Pearson Education, Inc.

[3] Nagy, Gabriel. 2012. Ordinary Differential Equations. Michigan State University: Mathematics Department.

[4] Swift, Randal J. dan Stephen A. Wirkus. 2007. A Course in Ordinary Differential Equations. Taylor \& Francis Group: Chapman \& Hall/CRC. 